\title{
Evolving spheres of shear-free anisotropic fluid
}

\author{
B.V.Ivanov \\ Institute for Nuclear Research and Nuclear Energy, \\ Bulgarian Academy of Science, \\ Tzarigradsko Shausse 72, Sofia 1784, Bulgaria
}

November 18, 2018

\begin{abstract}
The fluid models mentioned in the title are studied in a modified approach, based on two formulas for the mass function. All characteristics of the fluid are expressed through a master potential, satisfying an ordinary second order differential equation. Different constraints are imposed on this core of relations, finding new solutions and deriving the classical results for perfect fluids as particular cases. All charged anisotropic solutions, all conformally flat and all uniform density solutions are found. A large class of solutions with linear equation among the two pressures is derived, including the case of vanishing tangential pressure. The mechanism responsible for the appearance of equation of state is elucidated.
\end{abstract}

\section{Introduction}

The description of gravitational collapse and evolution of compact objects under various conditions remain among the important problems of general relativity. They are described by spherically symmetric relativistic fluid models where the metric depends on time and radius. In general, they possess shear, expansion and acceleration, which makes them rather hard to study. In many physical situations, however, shear-free perfect fluid models are a good approximation. The history of their study is long and rich in rediscoveries [1]. McVittie used an ad hoc ansatz to find a solution [2]. The solution 
of the Einstein equations may be reduced to a single ordinary second order differential equation [3], 4]. Its integration may be done by Lie point symmetry methods [4], [5], [6], [7, [8]. Another approach is to search for solutions which have the Painlevé property [9]. Other solutions have been found too [10].

The study of charged shear-free perfect fluids started with the papers of Shah and Vaidya [11] and Faulkes [12. The master equation acquires an additional non-derivative term. The three approaches mentioned above were applied to the charged case too finding new solutions [13], [14], [15], [16], [17], [18], [19], [20], 21]. It was noticed that both in the neutral and in the charged case the second Weyl invariant is tightly bound to the arbitrary functions in the master equation. Solutions which obey an equation of state (EOS) have been discussed in the neutral [3], [22] and the charged case [23], 24], 25], [26] and are known as the Wyman and the charged Wyman solution.

All perfect fluid solutions with uniform energy density have been found by Kustaanheimo [1], [27]. There are two branches - one in elementary functions and one given implicitly. Different properties of these solutions have been discussed [28], [29], [30], [31], [32], [33], [16], [34]. In some of these papers it was noticed that conformally flat solutions are particular cases of uniform density solutions. Charged shear-free perfect fluid spheres with uniform density are necessarily static [35]. As for the conformally flat perfect fluid solutions, all of them have been found by Stephani, using an embedding in a flat sixdimensional space [1], 36]. Some approximate conformally flat solutions for anisotropic fluid with slow motion were studied recently [37, as well as conformally flat solutions for radiating perfect fluids [38, [39]. Close to them are solutions admitting conformal symmetry [1], 40], [41].

On the other hand, different mechanisms have been identified through the years which create pressure anisotropy in stellar models and make the fluid imperfect [42]. Such are the exotic phase transitions during gravitational collapse, the existence of a solid core or the presence of a type $\mathrm{P}$ superfluid. Viscosity may also be a source of local anisotropy as well as the slow rotation of a fluid. It has been shown that the sum of two perfect fluids, two null fluids or a perfect and a null fluid may be represented by effective anisotropic fluid models [43. Recently it was pointed out that the same is true for perfect fluids with charge, bulk and shear viscosity [44].

The aim of the present paper is to generalize the above results, valid for shear-free perfect fluids, to shear-free anisotropic fluids. For this purpose we use the reformulation of the Einstein equations in terms of the mass function 
[45], [46]. It is combined with a formula for the same function derived recently [42], [47, [48] and adapted to our goals. In this approach, besides the new solutions, the classical results reviewed above follow as particular cases when the isotropy condition is imposed.

In Sec.2 we give a derivation of the mass decomposition formula in the general case of radiating spherically symmetric anisotropic fluid when both heat flow and null fluid are present. It consists of a local and a global part, the latter being the second Weyl invariant. In Sec.3 the field equations based on the mass function are given for non-radiating spherically symmetric metric. The fundamental potential $Z$ is introduced and a new formula for the shear is presented. Sec.4 discusses the general solution of the shear-free anisotropic model based on a single second order differential equation. In Sec.5 the particular cases of perfect fluids and charged anisotropic fluids are solved, making contact with the existing literature. In Sec.6 we impose in addition to the isotropy condition the requirement for EOS and rederive the Wyman and the charged Wyman solution. In Sec.7 the general conformally flat solution for anisotropic shear-free fluids is found and the classic Stephani solution is obtained in particular. The general uniform density solution is derived in

Sec. 8 and the known solution for perfect fluid is reobtained. A mechanism of producing models with special linear EOS is outlined in Sec.9. Sec.10 studies the case when the radial and the tangential pressures are proportional or one of them vanishes. Finally, some conclusions are drawn in the last section.

\section{Mass decomposition formula}

Spherically symmetric relativistic fluid models are described by the metric

$$
d s^{2}=e^{2 \nu} d t^{2}-e^{2 \lambda} d r^{2}-Y^{2}\left(d \theta^{2}+\sin ^{2} \theta d \varphi^{2}\right),
$$

where $\nu, \lambda$ and $Y$ are functions of $t$ and $r$ only. The spherical coordinates are numbered as $x^{0}=t, x^{1}=r, x^{2}=\theta, x^{3}=\varphi$. In general relativity one defines the Weyl tensor $C_{i j k l}$ through the Riemann tensor of the spacetime $R_{i j k l}$ as follows [4]

$$
R_{i j k l}=C_{i j k l}+\frac{1}{2}\left(g_{i k} R_{j l}+g_{j l} R_{i k}-g_{j k} R_{i l}-g_{i l} R_{j k}\right)-\frac{1}{6}\left(g_{i k} g_{j l}-g_{j k} g_{i l}\right) R .
$$


Here $R_{i j}$ is the Ricci tensor and $R$ is its trace, the Ricci scalar. The mass function $m$ is given by

$$
m=\frac{1}{2} Y R_{232}^{3} .
$$

We need the Einstein equations

$$
R_{i j}-\frac{1}{2} g_{i j} R=8 \pi T_{i j}
$$

where $T_{i j}$ is the energy-momentum tensor (EMT) and we have set $G=c=1$. For an anisotropic fluid model with heat flow one has [50]

$$
T_{i k}=\left(\rho+p_{t}\right) u_{i} u_{k}-p_{t} g_{i k}+\left(p_{r}-p_{t}\right) \chi_{i} \chi_{k}+q_{i} u_{k}+q_{k} u_{i} .
$$

Here $\rho$ is the energy density, $p_{r}$ is the radial pressure, $p_{t}$ is the tangential pressure, $u^{i}$ is the four-velocity of the fluid, $\chi^{i}$ is a unit spacelike vector along the radial direction and $q^{i}$ is the heat flux (in the radial direction too). They satisfy the relations

$$
u^{i} u_{i}=1, \quad \chi^{i} \chi_{i}=-1, \quad u^{i} \chi_{i}=0, \quad u^{i} q_{i}=0 .
$$

The coordinates are assumed to be comoving, hence

$$
u^{i}=e^{-\nu} \delta_{0}^{i}, \quad \chi^{i}=e^{-\lambda} \delta_{1}^{i}, \quad q^{i}=q e^{-\lambda} \delta_{1}^{i},
$$

where $q=q(r, t)$. The traces of Eqs $(4,5)$ give respectively

$$
R=-8 \pi T, \quad T=\rho-2 p_{t}-p_{r},
$$

$T$ being the trace of the EMT.

Now we are in position to rewrite the basic Eq (2) in terms of $T_{i j}$ instead of $R_{i j}$

$$
R_{i j k l}=C_{i j k l}-4 \pi\left(g_{j k} T_{i l}+g_{i l} T_{j k}-g_{i k} T_{j l}-g_{j l} T_{i k}\right)-\frac{8 \pi}{3}\left(g_{i k} g_{j l}-g_{j k} g_{i l}\right) T .
$$

Let us note that for non-trivial $u_{i}, \chi_{i}$ and $q_{i}, i=0$ or 1 . Then for $i, j, k, l$ equal to 2 or 3 the previous equation greatly simplifies

$$
R_{i j k l}=C_{i j k l}-\frac{8 \pi}{3}\left(\rho+p_{t}-p_{r}\right)\left(g_{i k} g_{j l}-g_{j k} g_{i l}\right) .
$$


This gives for the mass function

$$
m=\frac{4 \pi}{3} Y^{3}\left(\rho+p_{t}-p_{r}\right)+\frac{Y}{2} C_{232}^{3} .
$$

Note that the tangential pressure enters this formula thanks to the trace $T$, while the heat flow coefficient $q$ does not appear at all. A similar formula has been found by inspecting the field equations [47], where non-comoving system of coordinates has been used and the expression in the brackets reads $T_{0}^{0}+T_{1}^{1}-T_{2}^{2}$. In non-comoving coordinates the individual components depend also on the radial velocity of the moving observer. One can check, however, that in the cited combination this dependence is cancelled and it coincides with the bracket term from Eq (11). We have proved this formula without resorting to the explicit expressions for the field equations. It is consequence of the 'orthogonal' character of the four-vectors present in the EMT (pointing in the time and radial direction) with respect to $m$, defined in terms of tensor components along the two spherical angles $\theta$ and $\varphi$.

Formula (11) holds also when the basic anisotropic fluid radiates a null fluid with EMT

$$
T_{i j}^{N}=\varepsilon l_{i} l_{j},
$$

where $l^{i}$ is a null vector, $l^{i}=u^{i}+\chi^{i}$. One can show [44 that it adds to the basic $T_{i k}$ effective energy density, radial pressure and heat flow, all of them equal

$$
\rho^{N}=p_{r}^{N}=q^{N}=\varepsilon .
$$

This addition does not change Eq (11) or, put in other words, $\varepsilon$ (like $q$ ) does not appear in it directly.

Next we shall use the definition of the second Weyl invariant $\Psi_{2}$ in terms of the Weyl tensor for the spherically symmetric case [51]. It reads after a slight transformation

$$
2 \Psi_{2}=\frac{C_{2323}}{g_{22} g_{33}}=-Y^{-2} C^{3}{ }_{232} .
$$

Hence, one can rewrite formula (11) as

$$
m=\frac{4 \pi}{3} Y^{3}\left(\rho+p_{t}-p_{r}\right)-Y^{3} \Psi_{2} .
$$


In the static case one can set $Y=r$. For a perfect fluid $m$ contains only its energy density. In the general case the anisotropic factor $\Delta p=p_{t}-p_{r}$ is added. Thus, the total mass function is a sum of the local part arising directly from matter sources and the Weyl term, responsible for some global curvature. This last term may be related also to the electric part of the Weyl tensor 48

\section{$3 \quad$ Field equations}

From now on we accept that there is no radiation $(q=\varepsilon=0)$. We also change the signature of the metric. The usual field equations (4) are rather cumbersome except for the (01) component

$$
\dot{Y}^{\prime}-\dot{Y} \nu^{\prime}-Y^{\prime} \dot{\lambda}=0
$$

The dot above means time derivative, while the prime denotes a radial one. In order to make use of the mass decomposition formula (15) we shall work in the formalism based on $m$ [45], [46], namely

$$
\begin{gathered}
m^{\prime}=4 \pi \rho Y^{2} Y^{\prime}, \\
\dot{m}=-4 \pi p_{r} Y^{2} \dot{Y}, \\
\dot{\lambda}\left(\rho+p_{r}\right)=-\dot{\rho}-\frac{2 \dot{Y}}{Y}\left(\rho+p_{t}\right), \\
\nu^{\prime}\left(\rho+p_{r}\right)=-p_{r}^{\prime}+\frac{2 Y^{\prime}}{Y}\left(p_{t}-p_{r}\right) .
\end{gathered}
$$

The mass is given by Eq (3) which yields

$$
m=\frac{1}{2} Y\left(1+e^{-2 \nu} \dot{Y}^{2}-e^{-2 \lambda} Y^{\prime 2}\right) .
$$

The expansion of the fluid is

$$
\Theta=e^{-\nu}\left(\dot{\lambda}+\frac{2 \dot{Y}}{Y}\right),
$$

while the components of the shear tensor are proportional to

$$
\sigma=\frac{1}{3} e^{-\nu}\left(\frac{\dot{Y}}{Y}-\dot{\lambda}\right) .
$$


Let us introduce next a quantity $Z$, which is central in the following

$$
Z=\Psi_{2}-\frac{4 \pi}{3} \Delta p .
$$

Then the mass decomposition formula (15) may be written as

$$
\frac{4 \pi}{3} \rho=\frac{m}{Y^{3}}+Z \text {. }
$$

This is an expression for the energy density which, unlike Eq (17), contains the mass and not its derivative. Making use of Eq (17) we obtain

$$
m-\frac{m^{\prime} Y}{3 Y^{\prime}}=-Y^{3} Z
$$

or in a more compact form

$$
\left(\frac{m}{Y^{3}}\right)^{\prime} \frac{Y}{3 Y^{\prime}}=Z .
$$

Next we find another expression for the shear. Replacing $m$ from Eq (15) into $\mathrm{Eq}(18)$ gives

$$
3 \dot{Y}\left(\rho+p_{t}\right)=Y \dot{p}_{r}-Y\left(\dot{\rho}+\dot{p}_{t}\right)+\frac{3\left(Y^{3} \Psi_{2}\right)}{4 \pi Y^{2}} .
$$

Replacing in Eq (23) $\dot{\lambda}$ from Eq (19) and using the previous equation we find

$$
\left(\rho+p_{r}\right) \sigma=\frac{e^{-\nu}}{3 Y}\left[\frac{3\left(Y^{3} \Psi_{2}\right)^{\cdot}}{4 \pi Y^{2}}-(Y \Delta p)^{\cdot}\right] .
$$

The formulae in this section hold for any spherically symmetric metric and no radiation.

\section{Shear-free anisotropic fluids}

Let us impose on the metric the shear-free condition $\sigma=0$. Eq (23) can be integrated up to an arbitrary function of $r$. Usually it is chosen in such a way that

$$
e^{2 \lambda}=\frac{Y^{2}}{r^{2}} .
$$


Then Eq (16) is also integrable and determines $\nu$ up to an arbitrary function of time. Eq (22) shows that it is related to the expansion of the fluid and finally

$$
e^{2 \nu}=\frac{9}{\Theta^{2}} \frac{\dot{Y}^{2}}{Y^{2}}
$$

where $\Theta(t)$ is an arbitrary function [1].

In the following we shall use sometimes the notation

$$
L=\frac{r}{Y}, \quad x=r^{2} .
$$

The metric may be written in terms of $Y$ or $L$

$$
d s^{2}=-\frac{9}{\Theta^{2}} \frac{\dot{Y}^{2}}{Y^{2}} d t^{2}+\frac{Y^{2}}{r^{2}} d r^{2}+Y^{2} d \Omega^{2}=L^{-2}\left(-\frac{9}{\Theta^{2}} \dot{L}^{2} d t^{2}+d r^{2}+r^{2} d \Omega^{2}\right) .
$$

Here $d \Omega$ denotes the angular part. The same is true for Eq (21)

$$
m=\frac{1}{2} Y\left(1+\frac{\Theta^{2}}{9} Y^{2}-\frac{r^{2} Y^{\prime 2}}{Y^{2}}\right)=Y^{3}\left[\frac{\Theta^{2}}{18}+2 L_{x}\left(L-x L_{x}\right)\right],
$$

which leads to a formula for the density

$$
\frac{4 \pi}{3} \rho=\frac{\Theta^{2}}{18}+2 L_{x}\left(L-x L_{x}\right)+Z .
$$

Eq (27) simplifies considerably because of Eq (34) and becomes

$$
\frac{4}{3} x L L_{x x}=Z
$$

Let us utilize next the second formula for the shear, namely Eq (29). It supplies the equation

$$
\frac{3\left(Y^{3} \Psi_{2}\right)^{\cdot}}{4 \pi Y^{2}}-(Y \Delta p)^{\cdot}=0=\left(\frac{\Psi_{2}}{L^{3}}\right)^{\cdot} L^{2}-\frac{4 \pi}{3}\left(\frac{\Delta p}{L}\right)^{\cdot} .
$$

Combining it with the definition of $Z$ one expresses both $\Delta p$ and $\Psi_{2}$ through Z

$$
\frac{4 \pi}{3} \Delta p=-\frac{\left(Y^{3} Z\right)^{\cdot}}{2 Y^{2} \dot{Y}}=\frac{L^{4}}{2 \dot{L}}\left(\frac{Z}{L^{3}}\right)
$$




$$
\Psi_{2}=-\frac{(Y Z)^{\cdot}}{2 \dot{Y}}=\frac{L^{2}}{2 \dot{L}}\left(\frac{Z}{L}\right) .
$$

The radial pressure is determined from $\mathrm{Eq}(18)$ where the mass is given by $\mathrm{Eq}$ (34). Together with the above formula for the anisotropy factor and Eq (25) for the energy density they give two expressions for the tangential pressure

$$
4 \pi p_{t} Y^{2} \dot{Y}=-\left(m+\frac{3}{2} Y^{3} Z\right)=\frac{1}{2}\left(m-4 \pi \rho Y^{3}\right)
$$

In the general formalism $\rho$ and $p_{r}$ are expressed through the mass by the similar formulae $(17,18)$ while $p_{t}$ should be found either from Eq $(19)$ or Eq (20). For shear-free fluids, however, it is given by the above formula, similar in structure to the formula for the radial pressure.

Now we are in position to outline in two different ways the general solution for shear-free anisotropic fluids. First, we choose an arbitrary $L(r, t)$ and find the metric, the mass function and $Z$ according to Eqs $(33,34,36)$. Then the energy density, the pressures and the second Weyl invariant are given correspondingly by Eqs $(35,18,40,39)$. In the second way we take an arbitrary $Z(r, t)$ and solve for $L$ from $\mathrm{Eq}(36)$, promoting the integration constants to arbitrary functions of time. This, however, is rather difficult. For example,

if $Z$ is a power $x^{n+1}$, we get an Emden-Fowler equation which has explicit solutions for $n=0,-1,-2$ only [52]. Different additional constraints on $Z$ will be discussed in the following, leading to other solutions.

The shear-free condition (37) can be written in integral form too

$$
Z=-\frac{8 \pi}{3 Y^{3}} \int \Delta p Y^{2} \dot{Y} d t+\frac{g(r)}{Y^{3}}=\frac{8 \pi L^{3}}{3} \int \Delta p \frac{\dot{L}}{L^{4}} d t+h(x) L^{3}
$$

where $g, h$ are arbitrary functions of $r$ or $x$.

\section{Perfect fluids. Charging a fluid}

The arbitrary function in the general shear-free anisotropic fluid solution indicates that we are free to impose another condition. This can be done in a number of ways. As a first example we impose the isotropy condition $p_{r}=p_{t}$ which makes the fluid perfect. Hence, perfect fluid can be viewed upon as an anisotropic fluid with linear EOS among the pressures. Then the 
integral term in Eq (41) disappears and the central equation (36) becomes

$$
L_{x x}=F(x) L^{2}, \quad F(x)=\frac{3 h(x)}{4 x} .
$$

This equation is well-known [3], 4]. When $F=(x-a)^{n}$, where $a, n$ are constants, it becomes an Emden-Fowler equation and possesses five analytical solutions for $n=0,-15 / 7,-20 / 7,-5 / 2,-5[9]$, [52]. All of them may be expressed through the Weierstrass elliptic function. The known solutions of Eq (42) [1, 9, [10] include some others, like the second Painlevé transcendent. The time dependence appears in $L$ by turning the integration constants into integration functions of time. The general formulae give expressions for the other characteristics of the fluid and the freedom in the solution is reduced to arbitrary functions of time. Eq (24) shows that for $\Delta p=0$ we have $Z=\Psi_{2}$ and hence

$$
\Psi_{2}=\frac{4}{3} x F(x) L^{3}
$$

which too is a classical result [1], [16]. We see that the appearance of the second Weyl invariant is due to the mass decomposition formula, which in its turn stems from the definition of the Weyl tensor, applied to an interior fluid solution.

Another way to constrain the form of $\Delta p$ is to charge the fluid. Spherical symmetry allows the appearance of only an electric field $E$ in the radial direction. The energy-momentum tensor of this field can be described [44] as addition of effective energy density and pressures to the fluid with

$$
\rho^{E}=p_{t}^{E}=-p_{r}^{E}=\frac{E^{2}}{8 \pi}
$$

The Maxwell equations give

$$
\Delta p^{E}=\frac{E^{2}}{4 \pi}=f(x) L^{4}, \quad 4 \pi \tau=E e^{-\lambda},
$$

where $\tau$ is the charge function of the fluid and $f(x)$ is an arbitrary function. Hence, Eq (41) acquires a charge term in addition to other sources of anisotropy

$$
Z=h(x) L^{3}+\frac{8 \pi}{3} f(x) L^{4}+\frac{8 \pi}{3} L^{3} \int \Delta p \frac{\dot{L}}{L^{4}} d t
$$


In the case of charged perfect fluid Eq (36) becomes

$$
L_{x x}=\frac{3 h}{4 x} L^{2}+\frac{2 \pi f}{x} L^{3},
$$

which is also well-known and studied in the past [11], [12]. The Weyl invariant reads

$$
\Psi_{2}=h L^{3}+4 \pi f L^{4}
$$

and we have shown the reason for its close resemblance to $Z$. When

$$
h=x^{n_{1}+1}, \quad f=x^{n_{2}+1}
$$

$\mathrm{Eq}(47)$ has five different analytical solutions for $\left(n_{1}, n_{2}\right)=(0,0),(-5 / 2,-3)$, $(-14 / 5,-18 / 5),(-11 / 5,-12 / 5),(-5,-5)$ [52]. The density and the pressures are given by the general formulae plus the additions from Eq (44). In total, charged perfect fluid is a particular case of the anisotropic fluid model.

One can find new neutral anisotropic solutions by prescribing the form of the anisotropy factor. For example

$$
\Delta p=f(x) L^{a}, \quad L_{x x}=\frac{3 h}{4 x} L^{2}+\frac{2 \pi f}{(a-3) x} L^{a-1},
$$

where $a$ is some constant, not equal to 3 .

\section{Solutions with equation of state}

There is a case when the integration of $\mathrm{Eq}(47)$ is straightforward. Let the $x$-functions on the r.h.s become constants $\alpha, \beta$. Multiplication with $L_{x}$ gives a first integral

$$
L_{x}^{2}=\xi+\frac{2}{3} \alpha L^{3}+\frac{1}{2} \beta L^{4}
$$

where $\xi$ is another constant. Integrating once more one finds

$$
\int \frac{d L}{\sqrt{\frac{\beta}{2} L^{4}+\frac{2 \alpha}{3} L^{3}+\xi}}=x+t \equiv u,
$$

where an arbitrary function of time has been set to $t$. This is a particular form of the integral

$$
u=\int_{0}^{L} f(s)^{-1 / 2} d s, \quad f(s)=a_{0} s^{4}+4 a_{1} s^{3}+6 a_{2} s^{2}+4 a_{3} s+a_{4}
$$


and other examples of it will appear later. It may be inverted in terms of the Weierstrass function $P\left(u, g_{2}, g_{3}\right)$ [53], namely

$$
\begin{gathered}
L(u)=\frac{\sqrt{a_{4}} P_{u}+2 a_{3}\left(P-\frac{a_{2}}{2}\right)+a_{1} a_{4}}{2\left(P-\frac{a_{2}}{2}\right)^{2}-\frac{1}{2} a_{0} a_{4}}, \\
g_{2}=a_{0} a_{4}-4 a_{1} a_{3}+3 a_{2}^{2}, \\
g_{3}=a_{0} a_{2} a_{4}+2 a_{1} a_{2} a_{3}-a_{2}^{3}-a_{0} a_{3}^{2}-a_{1}^{2} a_{4} .
\end{gathered}
$$

Here $a_{i}$ are constants or functions of time. In the present case

$$
g_{2}=\frac{1}{2} \beta \xi, \quad g_{3}=-\frac{1}{36} \alpha^{2} \xi .
$$

Eq (35) shows that while $L=L(u)$ the density in general is $\rho(t, r)$ because of terms with $x$. However, Eqs $(44,45)$ lead to their cancellation and tuning the expansion yields

$$
8 \pi \rho(u)=3(C-4 \xi u)+12 L L_{u}, \quad \Theta^{2}=9 C-36 \xi t,
$$

where $C$ is a constant. Eq (19) holds for the effective energy and pressures of the charged perfect fluid. It gives the following equation for the sum of the genuine energy density and isotropic pressure

$$
8 \pi(\rho+p)=\frac{4 L^{4}}{L_{u}}\left(\frac{5}{3} \alpha+\frac{3}{2} \beta L\right) .
$$

Hence, $p=p(u)$ and $\rho=\rho(p)$. The fluid possesses an EOS and this is the charged Wyman solution [23], [24], [25], [26]. Combining the two previous equations we obtain

$$
8 \pi(\rho+6 p)=-15(C-4 \xi u)-60 \xi \frac{L}{L_{u}}+6 \beta \frac{L^{5}}{L_{u}} .
$$

When the fluid is neutral $(\xi=0, \beta=0)$ the EOS becomes linear and the Wyman solution emerges [3], 22].

In principle, the general solution of the shear-free anisotropic fluid depends on an arbitrary function $L(t, r)$. Going to a perfect or charged perfect fluid means imposing a differential equation on $L$ so that the freedom reduces to functions of $t$ or $r$. Imposing further an EOS makes the system overdetermined and solutions (if any) may exist only in some exceptional cases, like $\mathrm{Eq}(60)$, which holds for constant $\xi, \alpha, \beta$. 
Is it possible to generalize this EOS mechanism to anisotropy which is not the result of charging the fluid? For this purpose we take

$$
Z=\frac{4}{3} x L F(L)_{L}, \quad L_{x x}=F(L)_{L}, \quad L_{x}^{2}=2 F(L)+g(t),
$$

where $F(L)$ is an arbitrary function of $L$. The main equation still may be integrated and $L=L(u)$ if the integration function of time is constant $g_{0}$. Eq (35) gives

$$
4 \pi \rho=\frac{\Theta^{2}}{6}+6 L L_{u}+2 x\left(2 L F_{L}-6 F-3 g_{0}\right) .
$$

The elimination of the $x$-term requires an equation for $F$ leading to the Wyman solution. Thus, it is not possible to generalize it to anisotropic fluids except for a charged perfect fluid.

\section{Conformally flat solutions}

These solutions require $\Psi_{2}=0$. The definition of $Z$ and the vanishing shear constraint (37) then yield

$$
\Delta p=f(x) L, \quad Z=-\frac{4 \pi}{3} f(x) L, \quad L_{x x}=-\frac{\pi f(x)}{x},
$$

$f(x)$ being an arbitrary function. The differential equation is easily integrated so that

$$
L=G(x)+h(t) x+q(t), \quad f(x)=-\frac{1}{\pi} x G(x)_{x x}
$$

and $L$ depends on three arbitrary functions. The expressions for $m, \rho, p_{r}, p_{t}$ are rather cumbersome and are not given. Let us note a general equation which follows from Eqs $(18,40)$

$$
2 p_{t}+p_{r}+3 \rho=\frac{L}{\dot{L}} \dot{\rho} .
$$

Things simplify considerably for a conformally flat perfect fluid. Then one can put $G=0$ and Eqs $(15,35,64)$ give

$$
4 \pi \rho=\frac{\Theta(t)^{2}}{6}+6 h(t) q(t), \quad m=\frac{4 \pi r^{3} \rho}{3 L^{3}}
$$


so that $\rho$ is a function of time only. Eq (65) then gives for $p$

$$
p=-\rho+\frac{\dot{\rho} L}{3 \dot{L}} .
$$

This is exactly the Stephani solution [1], [36]. Eq (29) shows that when $\Psi_{2}=0=\Delta p$ the shear vanishes identically. Thus Stephani's solution describes all conformally flat perfect fluids, while the solution (64) describes all conformally flat anisotropic fluids with vanishing shear. Comparison between Eq (45) and Eq (63) shows that the anisotropy can not be caused by charging the fluid unless the solution is static, hence, evolving charged perfect fluid can not be conformally flat in addition. This result was found in the past by other methods [35].

\section{Uniform density}

In the previous section we have seen that some conformally flat solutions possess uniform density $\rho(t)$. One can find all solutions of this kind. Up to now we have worked in the $L$-formalism, taking $L$ as a basic function. From now on we shall work in the $Y$-formalism. For uniform energy density Eq (17) may be integrated

$$
m=\frac{4 \pi}{3} \rho Y^{3}-f(t)
$$

and Eqs $(27,38)$ give

$$
Z=\frac{f(t)}{Y^{3}}, \quad \frac{4 \pi}{3} \Delta p=-\frac{\dot{f}}{2 Y^{2} \dot{Y}}
$$

The combination of Eqs $(34,68)$ provides a first order differential equation for $Y$

$$
\begin{gathered}
Y_{z}^{2}=A Y^{4}+Y^{2}+2 f Y, \\
A(t)=\frac{\Theta^{2}}{9}-\frac{8 \pi}{3} \rho, \quad z=\ln r,
\end{gathered}
$$

which can be integrated

$$
\int \frac{d X}{\sqrt{2 f X^{3}+X^{2}+A}}=s, \quad X=\frac{1}{Y}, \quad s=\ln r B(t) .
$$


Here another arbitrary function of time appears. One can invert the functional dependence with the help of formula (54) and express $Y$ in terms of the Weierstrass function with

$$
g_{2}=\frac{1}{12}, \quad g_{3}=-\frac{1}{216}-\frac{f^{2} A}{4} .
$$

The pressures are obtained from Eqs $(65,69)$.

In the case of perfect fluid the function $f(t)$ becomes a constant and the above solution still applies. When this constant vanishes, $Z=0=\Psi_{2}$ and the solution is conformally flat. The integral above is expressed in elementary functions. We find

$$
r X=L=e^{-\lambda}=-\frac{A}{B}+\frac{B}{4} r^{2},
$$

which coincides with $\mathrm{Eq}(64)$ when $G=0$. These results are the same as the classical results on perfect fluids with uniform density [1, [27]. They can be rewritten in the $L$-formalism. There $L$ satisfies Eq (42) with $F(x) \sim x^{-5 / 2}$ which follows from $\mathrm{Eq}(69)$.

In the general anisotropic case one has $Y=Y(s)$ when $f$ and $A$ are constants. Then $\Delta p=0$ and the fluid is in fact perfect. Further, $m=m(s)$ when $\Theta$ is constant. This leads to the constancy of $\rho$ and $p$. Then Eqs (18, 68 ) show that $\rho+p=0$. Once again the fluid has an EOS when the arbitrary time functions become constants.

\section{Solutions with $\rho+p_{r}=0$}

We may generalize the uniform density case with the help of an arbitrary function $F(Y)$. Mimicking $\mathrm{Eq}(69)$, let us take

$$
Z=f(t) F(Y)_{Y} Y
$$

In particular, uniform density follows from $F=-1 / 3 Y^{3} . \mathrm{Eq}(27)$ gives the mass function

$$
\frac{m}{Y^{3}}=3 f(t) F(Y)+g(t)
$$

and then $\rho$ is found from $\mathrm{Eq}(25)$

$$
\frac{4 \pi}{3} \rho=f\left(3 F+Y F_{Y}\right)+g .
$$


Proceeding like in the previous section we obtain

$$
\int \frac{d X}{\sqrt{-6 f F\left(\frac{1}{X}\right)+X^{2}+A_{1}}}=s, \quad A_{1}(t)=\frac{\Theta^{2}}{9}-2 g,
$$

which determines $Y(s, t)$. We also find

$$
4 \pi\left(\rho+p_{r}\right)=-(3 \dot{f} F+\dot{g}) \frac{Y}{\dot{Y}}, \quad-\frac{8 \pi}{3} \Delta p=\dot{f} F_{Y} \frac{Y^{2}}{\dot{Y}}+4 Z,
$$

which determine the pressures. When $f(t), g(t)$ are constants we get a linear $\operatorname{EOS} \rho+p_{r}=0$. The density and pressures are not constant like in the previous section.

The appearance of EOS can be seen in another way too. We have $m=$ $m(Y)$ from $\mathrm{Eq}(76)$. Eqs $(17,18)$ indicate that in this case the difference between time and space derivatives effectively disappears and $\rho+p_{r}=0$. The discussion in the present section is analogous to that in Sec. 6 which lead to the Wyman solution. However, now we work in the $Y$-formalism and the EOS differs from Eq (60), reached in the $L$-formalism.

\section{Solutions with $p_{t}=\gamma p_{r}$ or $p_{r}=0$}

Solutions with vanishing tangential or radial pressure have been discussed in the past for time-dependent anisotropic fluids with shear [54], [55], [56]. We shall investigate the more general case of linear EOS between the pressures

$$
p_{t}=\gamma p_{r}
$$

Here $\gamma$ is some constant measuring the degree of anisotropy between the two extreme cases $\gamma=0\left(p_{t}=0\right)$ and $\gamma=1\left(p_{t}=p_{r}\right.$, isotropy condition). We have shown that for shear-free fluids $p_{t}$ satisfies Eq (40), similar in structure to $\mathrm{Eq}(18)$ for $p_{r}$. This allows to write Eq (80) as a time derivative and to integrate it, obtaining

$$
\frac{3}{2} Y^{3} Z+(1-\gamma) m=g(r),
$$

$g(r)$ being arbitrary. In addition, $Z$ satisfies $\mathrm{Eq}(26)$ for any spherically symmetric metric. This leads to

$$
\left(\frac{m}{Y^{1+2 \gamma}}\right)^{\prime}=\frac{2 g(r) Y^{\prime}}{Y^{2(1+\gamma)}}
$$


Suppose that $g(r)=g_{0}$, a constant. Then another integration gives

$$
m=a+k(t) Y^{1+2 \gamma}, \quad a=-\frac{2 g_{0}}{1+2 \gamma}
$$

and $k(t)$ is another arbitrary function. Eqs $(81,17)$ read

$$
\begin{gathered}
Z=-\frac{a}{Y^{3}}+\frac{2(\gamma-1)}{3} k Y^{2(\gamma-1)}, \\
4 \pi \rho=(1+2 \gamma) k Y^{2(\gamma-1)} .
\end{gathered}
$$

Eqs $(38,80)$ result in

$$
4 \pi p_{r}=-4 \pi \rho-\dot{k} \frac{Y^{2 \gamma-1}}{\dot{Y}},
$$

which allows to find $\Psi_{2}$ from the definition of $Z$

$$
\Psi_{2}=-\frac{a}{Y^{3}}+\frac{1}{3}(\gamma-1)(1-2 \gamma) k Y^{2(\gamma-1)}-\frac{1}{3}(\gamma-1) \dot{k} \frac{Y^{2 \gamma-1}}{\dot{Y}} .
$$

The combination of Eqs $(34,83)$ provides a first-order equation for $Y$ or $X=1 / Y$ which can be integrated

$$
\int \frac{d X}{\sqrt{-2 a X^{3}+X^{2}-2 k X^{2(1-\gamma)}+\Theta^{2} / 9}}=s .
$$

The integral may be inverted with the Weierstrass function when the polynomial $Q$ under the root is up to the fourth degree. This happens when $\gamma=0, \pm 1 / 2, \pm 1$.

In the case $\gamma=0\left(p_{t}=0\right)$ the polynomial and the other characteristics of the fluid read

$$
\begin{gathered}
Q=-2 a X^{3}+(1-2 k) X^{2}+\frac{\Theta^{2}}{9}, \\
m=a+k Y, \quad Z=-\frac{a}{Y^{3}}-\frac{2 k}{3 Y^{2}}, \\
4 \pi \rho=\frac{k}{Y^{2}}, \quad 4 \pi\left(\rho+p_{r}\right)=-\frac{\dot{k}}{Y \dot{Y}}, \\
\Psi_{2}=-\frac{a}{Y^{3}}+\frac{k}{3 Y^{2}}+\frac{\dot{k}}{3 Y \dot{Y}} .
\end{gathered}
$$


When $k$ is constant $\rho+p_{r}=0, m=m(Y), Z=Z(Y)$ and the solution belongs also to the class discussed in the previous section.

In the case $\gamma=1$ (perfect fluid) one finds

$$
\begin{gathered}
Q=-2 a X^{3}+X^{2}+\frac{\Theta^{2}}{9}-2 k, \\
m=a+k Y^{3}, \quad Z=-\frac{a}{Y^{3}}, \\
4 \pi \rho=3 k, \quad 4 \pi\left(\rho+p_{r}\right)=-\frac{\dot{k} Y}{\dot{Y}}, \quad \Psi_{2}=-\frac{a}{Y^{3}} .
\end{gathered}
$$

Now $\rho=\rho(t)$ and this is in fact a uniform density solution given by Eq (72) when $f(t)=-a$. When $a=0$ the solution is conformally flat as can readily be seen from Eq (95).

Let us discuss finally the case $p_{r}=0$. Eq (18) gives $m=m(z)$. Substituting this into Eq (34) yields

$$
X_{z}^{2}=-2 m(z) X^{3}+X^{2}+\frac{\Theta^{2}}{9} .
$$

This equation may be integrated easily when $m$ is constant and a formula close to Eq (72) or to Eq (88) with $Q$ from (89) or (93) is obtained. However, this is unphysical case because the energy density vanishes. Therefore Eq (96) should be solved by other methods.

\section{Conclusions}

Recently we have stressed the importance of anisotropic fluid models for astrophysics [44]. With the present paper we start their systematic study from the most general case. A decomposition of the mass function into a local and global part is redireved and related to the second Weyl invariant. It holds for any radiating anisotropic fluid which is spherically symmetric. Then, for simplicity, we turn off the radiation and combine this mass formula with the reformulation of the Einstein equations in terms of the mass function (given by a classic formula) and its derivatives. This results in the important relation (27) for the master potential $Z$ and another expression for the shear, $\mathrm{Eq}$ (29). Here the usual metric formula for the shear (23) is elevated to global and source quantities such as $\Psi_{2}$ and the anisotropy factor. It allows to push 
further the study of shear-free fluids, providing an important constraint. Now everything can be expressed in terms of $L$ and Eq (27) transforms into the second order Eq (36). The general solution of this equation is based on one arbitrary function which may be $Y, L, Z, \Delta p$ or $\Psi_{2}$. The treatment of the tangential pressure becomes on an equal footing with the radial pressure due to formula (40). The acceptance of maximum freedom in the system allows to obtain an anisotropic shear-free core of simple relations. Only after that we start to impose one by one different constraints aiming the system at particular cases.

Thus perfect fluid is this core with imposed isotropy condition $(\Delta p=0)$, charged fluid is a neutral fluid with a special kind of anisotropy, conformal flatness means the constraint $\Psi_{2}=0$, uniform density constrains the energy density to a function of time, anisotropy may be studied in more detail by a linear equation among the pressures, which includes the cases where one of them vanishes. In this way the relations among the particular cases become much clearer due to the relations of all of them to the core. Time and again second order equations like Eq (50) appear, which sometimes may be integrated to first order, like Eq (61) and even to implicit integral formulas for the solution, like Eqs $(52,72,78,88)$. These are different examples of the integral (53) which explains the persistent appearance of the Weierstrass elliptic function. In many cases, however, it reduces to elementary functions. This is true also for the $Y$-formalism.

Imposing one constraint fixes the arbitrary potential of the general model but up to functions of time or radius. Imposing a second constraint makes the system overdetermined, yet special solutions still exist when the arbitrary functions become constants. Then the integral formulas reduce the dependence on $t$ and $r$ to a dependence on a single variable $r^{2}+t$ or $r B(t)$. The result is appearance of equation of state, linear in some cases, as seen from Eqs $(60,79)$.

In total, we find the general charged anisotropic solution, all conformally flat solutions, all uniform density solutions, a large class of solutions with proportional or vanishing pressures and solutions with vanishing sum of density and radial pressure.

Finally, let us discuss the static case. Unfortunately, Eqs (16-21) are incomplete then because $(16,18,19)$ are trivial. The shear trivially vanishes and formula (31) holds no more. The metric function $\nu$ is not determined by $Y$ and becomes another free potential. One can use it and $\Delta p$ to express all static spherically symmetric anisotropic solutions [57]. 


\section{References}

[1] H. Stephani et al, Exact Solutions to Einstein's Field Equations (Cambridge University Press, 2003).

[2] G.C. McVittie, Mon. Not. R. Astron. Soc. 93, 325 (1933).

[3] M. Wyman, Phys. Rev. 70, 396 (1946).

[4] P. Kustaanheimo and B. Qvist, Comment. Phys. Math. Helsingf. 13, 16 (1948).

[5] H. Stephani, J. Phys. A: Math. Gen. 16, 3529 (1983).

[6] H. Stephani and T. Wolf, Class. Quantum Grav. 13, 1261 (1996).

[7] S. D. Maharaj, P.G.L. Leach and R. Maartens, Gen. Rel. Grav. 28, 35 (1996).

[8] C. Wafo Soh and F.M. Mahomed, Class. Quantum Grav. 16, 3553 (1999).

[9] M. Wyman, Canad. Math. Bull. 19, 343 (1976).

[10] D.C. Srivastava, Class. Quantum Grav. 4, 1093 (1987).

[11] Y.P. Shah and P.C. Vaidya, Tensor N.S. 19, 191 (1968).

[12] M.C. Faulkes, Can. J. Phys. 47, 1989 (1969).

[13] B. Mashhoon and M.H. Partovi, Phys. Rev. D 20, 2455 (1979).

[14] S. Chatterjee, Gen. Rel. Grav. 16, 381 (1984).

[15] R.A. Sussman, J. Math. Phys. 28, 1118 (1987).

[16] D.C. Srivastava, Fortschr. Phys. 40, 31 (1992).

[17] C. Wafo Soh and F.M. Mahomed, Class. Quantum Grav. 17, 3063 (2000).

[18] R. Halburd, Class. Quantum Grav. 18, 11 (2001).

[19] R.G. Halburd, J. Math. Phys. 43, 1966 (2002). 
[20] Y.K. Gupta and N. Bijalwan, Gen. Rel. Grav. 37, 1911 (2005).

[21] N. Bijalwan, Gen. Rel. Grav. 38, 981 (2006).

[22] D.C. Srivastava and S.S. Prasad, Gen. Rel. Grav. 15, 65 (1983).

[23] B. Mashhoon and M.H. Partovi, Ann. Phys. (N.Y.) 130, 99 (1980).

[24] B. Mashhoon and M.H. Partovi, Phys. Rev. D 30, 1839 (1984).

[25] D.C. Srivastava, Gen. Rel. Grav. 18, 1159 (1986).

[26] D.C. Srivastava, Class. Quantum Grav. 8, 1001 (1991).

[27] P. Kustaanheimo, Comment. Phys. Math. Helsingf. 13, 12 (1947).

[28] H. Bondi, Proc. R. Soc. A 281, 39 (1964).

[29] I.H. Thomson and G.J. Whitrow, Mon. Not. R. Astron. Soc. 136, 207 (1967).

[30] I.H. Thomson and G.J. Whitrow, Mon. Not. R. Astron. Soc. 139, 499 (1968).

[31] A. Banerjee, J. Phys. A: Gen. Phys. 5, 1305 (1972).

[32] J. Krishna Rao, Gen. Rel. Grav. 5, 351 (1973).

[33] P.S. Wesson and J. Ponce de Leon, Phys. Rev. D 39, 420 (1989).

[34] E. Brinis, S. Jhingan and G.Magli, Class. Quantum Grav. 17, 4481 (2000).

[35] R.M. Misra and D.C. Srivastava, Phys. Rev. D 8, 1653 (1973).

[36] H. Stephani, Commun. Math. Phys. 4, 137 (1967).

[37] L. Herrera, A. Di Prisco, J. Ospino and E. Fuenmayor, J. Math. Phys. 42, 2129 (2001).

[38] L. Herrera, G. Le Denmat, N.O. Santos and A. Wang, Int. J. Mod. Phys. D 13, 583 (2004).

[39] S.D. Maharaj and M. Govender, Int. J. Mod. Phys. D 14, 667 (2005). 
[40] S.D. Maharaj, P.G.L. Leach and R. Maartens, Gen. Rel. Grav. 23, 261 (1991).

[41] P. Havas, Gen. Rel. Grav. 24, 599 (1992).

[42] L. Herrera and N. O. Santos, Phys. Rep. 286, 53 (1997).

[43] P.S. Letelier, Phys. Rev. D 22, 807 (1980).

[44] B.V. Ivanov, Int. J. Theor. Phys. 49, 1236 (2010).

[45] G Lemaitre (republished), Gen. Rel. Grav. 29, 641 (1997) .

[46] M. E. Cahill and G. C. McVittie, J. Math. Phys. 11, 1382 (1970).

[47] L. Herrera, A. Di Prisco, J. L. Hernandez-Pastora and N. O. Santos, Phys. Lett. A 237, 113 (1998).

[48] L. Herrera, J. Ospino, A. Di Prisco, E. Fuenmayor and O. Troconis, Phys. Rev. D 79, 064025 (2009).

[49] S. Chandrasekhar, The Mathematical Theory of Black Holes (Oxford University Press, 1983).

[50] L. Herrera, N. O. Santos and A. Wang, Phys. Rev. D 78, 084026 (2008).

[51] B. Mashhoon and M. Hossein Partovi, Phys. Rev. D 20, 2455 (1979).

[52] A.D. Polyanin and V.F. Zaitsev, Handbook of Exact Solutions for Ordinary Differential Equations (Boca Raton, CRC Press, 1995).

[53] E.T. Whittaker and G.N. Watson, A Course of Modern Analysis (Cambridge University Press, 1927).

[54] C. Leibovitz, Phys. Rev. D 4, 2949 (1971).

[55] T.P. Singh and L. Witten, Class. Quantum Grav. 14, 3489 (1997).

[56] S.M.C.V. Goncalves and S. Jhingan, Gen. Rel. Grav. 33, 2125 (2001).

[57] L. Herrera, J. Ospino and A. Di Prisco, Phys. Rev. D 77, 027502 (2008). 